\title{
Morphology of Endothelial Cells from Different Regions of the Swine Cornea
}

\author{
Lídia Luzia Clerot, Paula Stieven Hünning, Marcelle Bettio, Marcela Torikachvili, Michelle Becker Petersen, \\ Alessandra Fernandez da Silva, Andre Silva Carissimi \& João Antonio Tadeu Pigatto
}

\begin{abstract}
Background: The corneal endothelium is a monolayer of polygonal cells which constitute the last layer of the cornea. The integrity of this layer is critical to cornea transparency. The characterization of normal corneal endothelial morphology is important not only to clinical evaluation but also to selection of areas of the cornea with better quality to be employed as donor tissue. The aim of the present study was to evaluate the morphology of endothelial cells from different regions of the swine cornea after alizarin red staining using optical microscopy. Materials, Methods \& Results: Twenty-four healthy eyes from 12 swine Large White breed, with 14-monthold, males or females obtained from a licensed Brazilian commercial slaughterhouse were studied. Immediately after humane slaughter, the eyes were enucleated and submitted to ophthalmic examination. Eyes with signs of diseases of the anterior segment were excluded. The cornea, with $3 \mathrm{~mm}$ of the sclera, was removed and placed on a glass microscope slide with the endothelial side up. Four radial incisions were made in the periphery of the cornea to better accommodate the cornea on the microscope slide. Alizarin red was diluted in isotonic solution $(0.2 \mathrm{~g} / 100 \mathrm{~mL})$ and the $\mathrm{pH}$ was adjusted to 4.2 with hydrochloric acid. Three drops of alizarin red were placed on the corneal endothelium. After $90 \mathrm{~s}$, the dye was removed from the cornea with balanced saline solution. The corneal endothelium was examined and photographed using an optical microscope. All evaluations were performed by the same investigator. Photomicrographs were taken of central, superior, inferior, nasal and temporal corneal areas. Parameter studied included endothelial cell morphology. For the statistical analysis, was employed the ANOVA variance test (repeated measures). Differences were considered statistically significant at $P<0.05$. Normal endothelium cells were mainly hexagonal $(83.7 \%)$, pentagonal $(7.45 \%)$ and heptagonal $(8.8 \%)$, with a minimal number of cells of other shapes present. There were no significant statistical differences in the proportion of the morphology and the different regions of the cornea $(P=0.31)$.

Discussion: Different techniques are available for the analysis of corneal endothelium, including mainly scanning electron microscopy, specular microscopy and optical microscopy. The analysis of the morphology of corneal endothelium with an optic microscope after staining with alizarin red has been described as an effective, rapid and cost-efficient method, since this dye blends the borated cells, allowing identification. In the present study, using optical microscopy and coloration with alizarin red it was possible to explore and to obtain images of the swine endothelium of all regions of the cornea. The analysis of the cellular morphology or the percentage of hexagonal cells are among the main parameters used to evaluate the health of the corneal endothelium. In this study, the endothelium had the predominance of the hexagonal shape in all regions studied. In swine, there are no studies evaluating the shape of the endothelial cells in the five different regions of the cornea. This study has demonstrated that the parameters evaluated in swine did not differ significantly between the various places of the cornea.
\end{abstract}

Keywords: pigs, corneal endothelium, alizarin red, pleomorphism. 


\section{INTRODUCTION}

The pump and barrier functions performed by the endothelium are vital for corneal transparency [19]. In many species, endothelial cells in the cornea have a low proliferative capacity, and lost cells are replaced by hypertrophy of adjacent cells, resulting in an increase size and shape variation these cell. Among the main methods used for endothelial analysis are scanning electron microscopy (SEM), specular microscopy and confocal micrscopy $[3,6,14,15]$. Is possible to analyze and photograph the endothelium of the cornea under an optical microscope after staining with alizarin red dye $[5,10,18,19]$.

Important studies have been performed regarding the description of the corneal endothelium of swine $[9,22]$. However, it is still unclear whether there is a difference in the endothelial morphology of the different corneal regions of this species. Knowledge of standard normal corneal endothelial cells in swine is important for understand the physiology of corneal of this species, which has been increasingly used as an experimental model in ophthalmology. Endothelial analysis usually includes assessment of the cell density, cell area, polymegathism and pleomorphism $[1,4]$.

Pig corneal diameter, thickness and refractive power have been compared with those of human corneas, with a demonstrated similarity between these species [8]. Such information about endothelial morphology will be important for future clinical studies in pigs using in-vivo techniques, and for research experiments. The purpose of this study was to assess the morphology of endothelial cells from different regions of the swine cornea after alizarin red staining using optical microscopy.

\section{MATERIALS AND METHODS}

\section{Samples}

Twenty-four healthy eyes from 12 swine Large White breed, with 14-month-old, males or females obtained from a licensed Brazilian commercial slaughterhouse (Salvadori abbatoir, Roca Sales, RS, Brazil) were studied. These pigs are slaughtered for reasons not related to this study. All procedures were performed in accordance with the Association for Research in Vision and Ophthalmology (ARVO) statement regarding the use of animals in ophthalmic and vision research.

\section{Methodology}

Immediately after humane slaughter, the eyes were enucleated and submitted to ophthalmic examina- tion, including biomicroscopy with a portable slit lamp SL $15^{1}$ and fluorescein staining ${ }^{2}$. Eyes that showed evidence of ocular disease were excluded. The cornea, with $3 \mathrm{~mm}$ of the sclera, was removed and placed on a glass microscope slide with the endothelial side up. Four radial incisions were made in the periphery of the cornea to better accommodate the cornea on the microscope slide. Alizarin red $^{3}$ was diluted in isotonic solution $(0.2 \mathrm{~g} / 100 \mathrm{~mL})$ and the $\mathrm{pH}$ was adjusted to 4.2 with hydrochloric acid. Three drops of alizarin red were placed on the corneal endothelium. After $90 \mathrm{~s}$, the dye was removed from the cornea with balanced saline solution. The corneal endothelium was examined and photographed using an optical microscope ${ }^{4}$. Five images were randomly captured from the central, upper, lower, temporal and nasal regions of the cornea. Morphological analysis of 100 endothelial cells from each region was performed with X 40 magnification. Microsoft's paint software was used in image processing. For the statistical analysis, was employed the ANOVA variance test (repeated measures). Differences were considered statistically significant at $P<0.05$.

\section{RESULTS}

The used technique enabled us to analyse the endothelium and to obtain images of the swine endothelium of all regions of the cornea. Polygonal, uniform and continuous cells were observed in all studied regions (Figure 1). Normal endothelium cells were mainly hexagonal $(83.7 \%)$, pentagonal $(7.45 \%)$ and heptagonal $(8.8 \%)$, with a minimal number of cells of other shapes present. The average percentage of hexagonal cells in the central area was $82.8 \pm 4.4 \%$; in the superior area, it was $82.7 \pm 4.6 \%$, in the inferior area, it was $84.2 \pm 5.2 \%$; in the temporal area, it was $85.0 \pm 3.6 \%$; and in the nasal area, it was $83.8 \pm 4.2 \%$ $(P=0.34)$. The average percentage of cells with five sides was $7.7 \pm 3.0 \%$ in the central area, $7.6 \pm 2.3 \%$ in the superior area, $7.56 \pm 3.1 \%$ in the inferior area, 7.5 $\pm 3.2 \%$ in the temporal area and $7.5 \pm 4 \%$ in the nasal area $(P=0.82)$. The average percentage of cells with seven sides was $9.55 \pm 3.2 \%$ in the central area, 9.55 $\pm 2.9 \%$ in the superior area, $8.3 \pm 2.5 \%$ in the inferior area, $8.0 \pm 3.6 \%$ in the temporal area and $8.6 \pm 3.3 \%$ in the nasal area $(P=0.82)$. There were no significant statistical differences in the proportion of the morphology and the different regions of the cornea $(P=0.31)$. The difference between the left and right ocular globes was not statistically significant. 


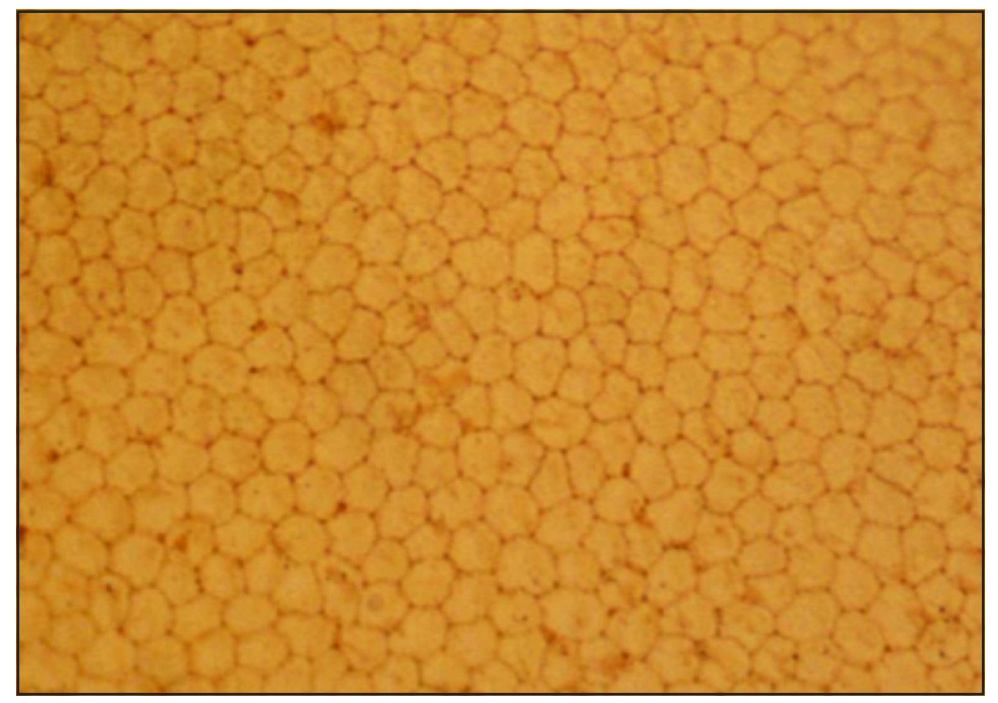

Figure 1. Optical photomicrograph of the pig corneal endothelium after staining with alizarin red [400x].

\section{DISCUSSION}

Several techniques are available for the analysis of corneal endothelium, including mainly SEM, specular microscopy and optical microscopy $[4,11,12,20]$. Currently, specular microscopy is the technique most used for clinical evaluations in humans and animals and for the analysis of surgical risks, mainly in cataract surgery $[1-3,6,14,15]$. Among the limitations of specular microscopy, difficulties in obtaining images in corneas with oedema and in capturing images of peripheral regions of the cornea have been mentioned [19]. When the cornea is not transparent, in vitro techniques have been employed for endothelial analysis, achieving good results [11$13,16,24]$. In the present study, coloration and optical microscopy enabled the analysis, photography and the study of the shape of the endothelium in all regions of the cornea. This methodology using optical microscopy and vital dyes could provide valuable data in corneas whose lack of clarity limits or precludes specular microscopy [7]. This technique had been performed previously with different concentrations of alizarin red dye. Previous studies compared the use of alizarin in the concentrations of 1 and $0.2 \%$ and found that the lower concentration marked the cell edges, with a higher action time of under endothelium at goat cornea [10]. In the present study, alizarin red dye was used at a concentration of $0.2 \%$ and enabled us to obtain clear images of the endothelial mosaic of all regions of the cornea. In addition, endothelial cell borders were visualised, allowing the quantification of the number of sides of each endothelial cell. This methodology enabled the evaluation of the effects of drugs and surgical procedures on the endothelium and the analysis of different means of preserving the cornea and the endothelial cell morphology of a healthy cornea $[5,20]$.

The preparation and use of alizarin red was based on previous studies, which used the dye to aid in the identification of endothelial damage or to study the cell morphology $[5,10,18,19,23]$. The analysis of corneal endothelium with an optic microscope after staining with alizarin red and trypan blue has been described as an effective, rapid and cost-efficient method [5,19]. For the quantification of areas of endothelial loss, it is necessary to use, in addition to alizarin red, trypan blue dye $[19,23]$. When the objective is to analyse only the endothelial morphology, alizarin red is sufficient, since this dye blends the borated cells, allowing identification [5]. In the present study, using only alizarin red dye it was possible to analyse the endothelium and to obtain images of the swine endothelium of all regions of the cornea.

Endothelial morphology and cell density are the most commonly used parameters in the assessment of corneal endothelium in humans and animals $[1,3,4,6]$. Due to the similarities to human eyes, swine eyes have been used extensively in experimental research $[9,17,21]$. The ocular bulbs and cornea sizes of 
pigs, their morphological endothelium characteristics, their wide availability and their low prices are some of the largest advantages of this choice in human and veterinary medicine [17]. Moreover, it has recently been suggested that young porcine eyes may be a great alternative to the transplantation of endothelial cells $[8,9]$.

In the present study, the use of eyes of animals from slaughterhouse allowed the standardisation of the sample with respect to age and the selection of healthy eyes. It also avoided the use of animals solely for research purposes. This methodology has already been used in previous studies, where it was possible to analyse the corneal endothelium within 6 hours after death without alterations in the ultrastructure of this layer $[2,5,6,11,13,15,16]$.

The analysis of the cellular morphology or the percentage of hexagonal cells is among the main parameters used to evaluate the health of the corneal endothelium $[4,11-13,15]$. At present, there is no consensus regarding the minimum number of cells that should be analysed to obtain maximum reliability in the analysed sample. In previous studies, the number of cells analysed in each cornea ranged from 30 to 100 [3]. In addition, when the specular microscope is used for endothelial analysis, the use of software for data reliability and reproducibility has been suggested [1]. In the present study, to reduce sampling and analysis errors, 100 endothelial cells were manually counted in each region of the cornea studied.

In the current study, the corneal endothelium consisted of polygonal cells, with predominance of the hexagonal shape in all regions studied. In addition, cells with five and seven sides were found. In both humans and in all species where the endothelium has been studied with respect to morphology normal corneal endothelium consists predominantly of hexagonal cells $[9,15]$. Previous searches, assessing the corneal endothelium of pigs, established by SEM that most of the cells were hexagonal [22]. Similarly, other researchers evaluated 12 months old swine eyes and found five-sided (18\%), six-sided (68\%) and seven-sided (14\%) cells [24]. For endothelial analysis, these authors used specular contact microscopy and analysed only the central region of the cornea. In the present study, the swine were 2 months old, and the percentage of hexagonal cells found was higher than that found by Terzariol and co-authors.
A previous study carried out in humans showed that with increasing age, the endothelial morphology becomes less regular and the percentage of cells with hexagonal shape decreases. Other researchers studied endothelial morphology in swine from three different age groups. The authors concluded that unlike humans, there was no significant correlation between percentage of hexagonal cells and age [9]. Regarding the endothelial morphology in swine, there are no studies evaluating the shape of the cells in the five different regions of the cornea. In the present study, we demonstrated that the parameters evaluated did not differ significantly between the different regions of the cornea. Previous studies in humans and animals have shown controversial results when endothelial parameters were compared in different regions of a healthy cornea [3,22].

Normally, in a healthy cornea, there is no difference in terms of endothelial parameters when the left eye is compared with the right eye $[2,5,6,14]$. The results obtained in the present study were consistent with those reported previously, where the endothelial parameters were similar in the left and the right eye.

Some researchers compared the values of the endothelial parameters obtained with light microscopy and specular microscopy. The result of this study indicated that considerable cell shrinkage occurs in rabbit endothelia following staining. Despite these significant changes in cell area, no significant alterations in cell shape occur. The percentage of hexagonal cells remains unchanged [7].

\section{CONCLUSION}

In accordance with the presented results the obtained values in relation to the shape of the cells of the endothelium at the central region might be extrapolated to the peripheral regions of healthy swine corneas.

\author{
MANUFACTURERS \\ ${ }^{1}$ Kowa Company. Tokyo, Japan. \\ ${ }^{2}$ Ophthalmos S.A. São Paulo, SP, Brazil. \\ ${ }^{3}$ Sigma Aldrich. St. Louis, MO, USA. \\ ${ }^{4}$ Nikon Instruments - Products. Tokyo, Japan.
}

Acknowledgements. The authors would like to thank Salvadori slaughterhouse for supplying the eyes used in this research.

Declaration of interest. The authors report no conflicts of interest. The authors alone are responsible for the content and writing of paper. 


\section{REFERENCES}

1 Abib F.C., Holzchuh R., Schaefer A., Schaefer T. \& Godois R. 2012. The endothelial sample size analysis in corneal specular microscopy clinical examinations. Cornea. 31(5): 546-550.

2 Albuquerque L., Freitas L.V.R. \& Pigatto J.A.T. 2015. Analysis of the corneal endothelium in eyes of chickens using contact specular microscopy. Semina: Ciências Agrárias. 36(6): 4199-4206.

3 Bercht B.S., Albuquerque L., Araujo A.C. \& Pigatto J.A.T. 2015. Specular microscopy to determine corneal endothelial cell morphology and morphometry in chinchilas (Chinchilla lanigera) in vivo. Veterinary Ophthalmology. 18(1): 137-142.

4 Coyo N., Penã M.T., Costa D., Ríos J., Lacerda R. \& Leiva M. 2015. Effects of age and breed on corneal thickness, density, and morphology of corneal endothelial cells in enucleated sheep eyes. Veterinary Ophthalmology. 19(5): 367-372.

5 Faganello C.S., Silva V.R.M., Andrade M.C.C., Carissimi A.S. \& Pigatto J.A.T. 2016. Morphology of endothelial cells from different regions of the equine cornea. Ciência Rural. 46(12): 2223-2228.

6 Franzen A.A., Pigatto J.A.T., Abib F.C., Albuquerque L. \& Laus J.L. 2010. Use of specular microscopy to determine corneal endothelial cell morphology and morphometry in enucleated cat eyes. Veterinary Ophthalmology. 13(4): 222-226.

7 Geroski D.H. \& Edelhauser H.F. 1989. Morphometric analysis of the corneal endothelium. Specular microscopy vs. alizarin red staining. Investigative Ophthalmology \& Visual Science. 30(2): 254-259.

8 Hara H. \& Cooper D.K.C. 2011. Xenotransplantation - the future of corneal transplantation? Cornea. 30(4): 371-378.

9 Lee S.E., Mehra R., Fujita M., Roh D.S., Long C., Lee W., Funderburgh J.L., Ayares D.L., Cooper D.K. \& Hara H. 2014. Characterization of porcine corneal endothelium for xenotransplantation. Seminars Ophthalmology. 29(3): 127-135.

10 Park S., Fong A.G., Cho H., Zhang C., Gritz D.C., Mian G., Herzlich A.A., Gore P., Morganti A. \& Chuck R.S. 2012. Protocol for vital dye staining of corneal endothelial cells. Cornea. 31(12): 1476-1479.

11 Pigatto J.A.T., Andrade M.C., Laus J.L., Santos J.M., Brooks D.E., Guedes P.M. \& Barros P.M.S. 2004. Morphometric analysis of the corneal endothelium of yacare caiman (Caiman yacare) using scanning electron microscopy. Veterinary Ophthalmology. 7(3): 205-208.

12 Pigatto J.A.T., Abib F. C., Pizzeti J.C., Laus J.L., Santos J. M. \& Barros P.S.M. 2005. Análise morfométrica do endotélio corneano de coelhos à microscopia eletrônica de varredura. Acta Scientiae Veterinariae. 33(1): 41-45.

13 Pigatto J.A.T., Laus J.L., Santos J.M., Cerva C., Cunha L.S., Ruoppolo V. \& Barros P.S.M. 2005. Corneal endothelium of the magellanic penguin (Spheniscus magellanicus) by scanning electron microscopy. Journal of Zoo and Wildlife Medicine. 36(4): 702-705.

14 Pigatto J.A.T., Abib F.C., Pereira G.T., Barros P.S.M., Freire C.D. \& Laus J.L. 2006. Density of corneal endothelial cells in eyes of dogs using specular microscopy. Brazilian Journal of Veterinary Research and Animal Science. 43(4): 476-480.

15 Pigatto J.A.T., Cerva C., Freire C.D., Abib F.C., Bellini L.P., Barros P.S.M. \& Laus J.L. 2008. Morphological analysis of the corneal endothelium in eyes of dogs using specular microscopy. Pesquisa Veterinária Brasileira. 28(9): 427- 430.

16 Pigatto J.A.T., Franzen A.A., Pereira F.Q., Almeida A.C.V.R., Laus J.L., Santos J.M., Guedes P.M. \& Barros P.S.M. 2009. Scanning electron microscopy of the corneal endothelium of ostrich. Ciência Rural. 39(3): 926-929.

17 Proulx S. \& Brunette I. 2012. Methods being developed for preparation, delivery and transplantation of a tissueengineered corneal endothelium. Experimental Eye Research. 95(1): 68-75.

18 Ruggeri A., Scarpa F., Massimo L., Meltendorf C. \& Schroeter J. 2009. A system for the automatic estimation of morphometric parameters of corneal endothelium in alizarine red-stained images. British Journal of Ophthalmology. 94(5): 643-647.

19 Saad H.A., Terry M.A., Shamie N., Chen E.S., Friend D.F., Holiman J.D. \& Stoeger C. 2008. An easy and inexpensive method for quantitative analysis of endothelial damage by using vital dying staining and adobe photoshop software. Cornea. 27(7): 818-824.

20 Silva V.R.M., Andrade M.C.C., Faganello C.S., Torikachvili M., Carissimi A.S. \& Pigatto J.A.T. 2018. Evaluation of equine corneal endothelium after exposure to $0.5 \%$ indocyanine green - in vitro study. Semina: Ciências Agrárias. 39(2): 613-620. 
21 Smeringaiova I., Merjava S.R., Stranak Z., Studeny P., Bednar J. \& Jirsova K. 2018. Endothelial wound repair of the organ-cultured porcine cornea. Current Eye Research. 43(7): 856-865.

22 Tamayo-Arango L.J., Baraldi-Artoni S.M., Laus J.L., Mendes-Vicenti F.A., Pigatto J.A.T. \& Abib F.C. 2009. Ultrastructure, morphology and morphometry of a normal corneal endothelium of adult crossbred pig. Ciência Rural. 9(1): 117-122.

23 Taylor M.L. \& Hunt C.J. 1981. Dual staining of corneal endothelium with trypan blue and alizarin red S: importance of $\mathrm{pH}$ for the dye-lake reaction. British Journal of Ophthalmology. 65(12): 815- 819.

24 Terzariol M., Hünning P.S., Brambatti G., Albuquerque L., Neumann C. \& Pigatto J.A.T. 2016. Effects of intracameral brilliant blue on the corneal endothelium of swine: in vitro study. Pesquisa Veterinária Brasileira. 36(8): 775-780. 